\title{
Recent Applications of the Gated Mesoporous Silica Nanoparticles as Drug Delivery System for Cancer Therapy: pH Sensitive Controlled Release
}

\section{Pervin Deveci*}

Department of Chemistry, Selcuk University, Turkey

*Corresponding author: Pervin Deveci, Selcuk University, Faculty of Science, Department of Chemistry, Konya, Turkey

\begin{abstract}
Mesoporous silica nanoparticles (MSNs) have attracted extensive attention as efficient drug delivery carriers on account of its adjustable pore size, specific surface area, stability, and good biocompatibility. This review summarizes the recent advances on the application of gated MSNs for controlled drug delivery. These advanced technologies demonstrate current challenges and provide a bright future for precision of cancer treatment.
\end{abstract}

Keywords: Hydrazone linker; Acetal linker; Cancer therapy; Imine linker; Controlled release; Stimuli-responsive

\section{Introduction}

Silica nanoparticles show desirable properties to be implemented as multifunctional nanoplatforms for drug delivery purposes, due to their unique characteristics, comprising extensive surface area, large pore size, lack of toxicity, biocompatibility, biodegradability, uniform distribution of target molecules on the

\section{Hydrazone Linker}

porous space, controllable superficial charge and free dissemination in the whole body [1-3]. This review intends to comprehensively analyses published papers that have used $\mathrm{pH}$ cleavable linkers (hydrazone linker, acetal linker, imine linker and boronic-ester linker) in combination with MSNs to design gated systems for controlled drug delivery applications.
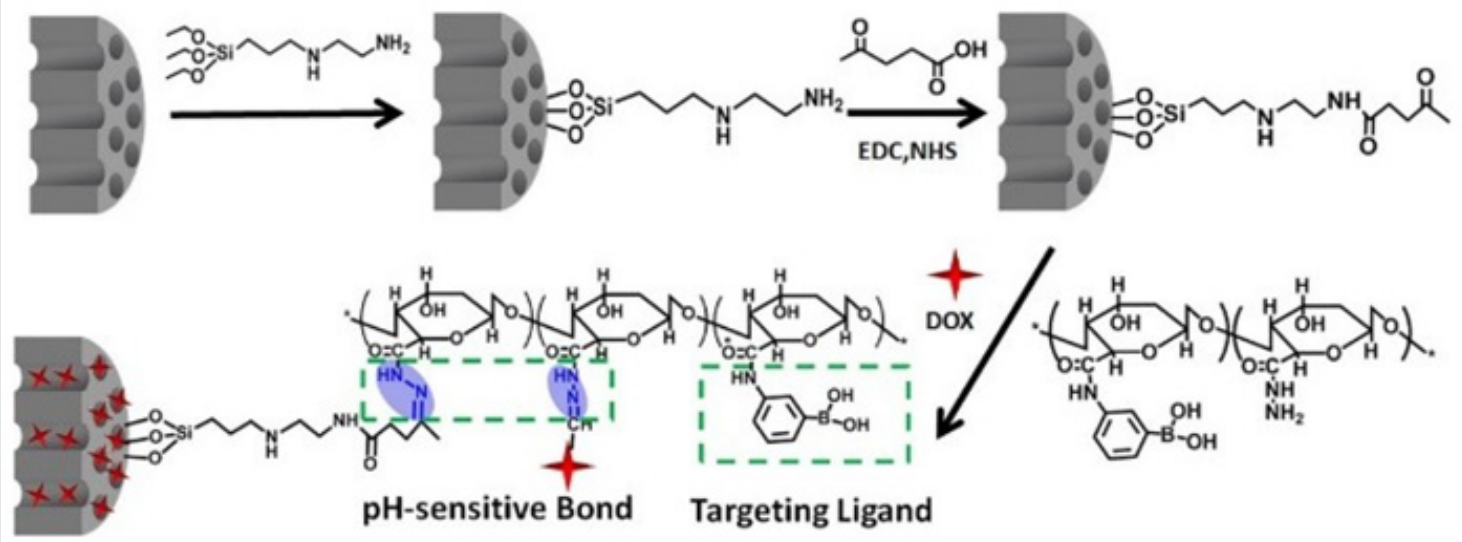

Figure 1: Schematic illustration of the fabrication of the Ph responsive targeted delivery system [4]. 
The hydrazone bond which is stable at physiological $\mathrm{pH}$ could undergo hydrolysis in an acidic environment, has been widely used to prepare smart drug delivery systems. Zhang and coworkers [4] developed multiple functional capping agents, which integrated pore-capping, drug-loading, and tumor-targeting abilities. As depicted in Figure 1, carbonyl was introduced on the surface of MSNs and a one-pot method was employed to load the chemotherapeutic agent in the pores and linked multifunctional capping agents via an acid-cleavable hydrazone bond at the same time. The drug-loading content of this delivery system can be as high as $10.6 \%$, but there was only $7.8 \%$ of drug leakage in three days in the blood circulation. Cui et al. [5] introduced $\mathrm{Fe}_{3} \mathrm{O}_{4}$ nanoparticles as a core coating with a mesoporous silica shell to obtain $\mathrm{Fe}_{3} \mathrm{O}_{4} @$ mesoporous silica $\left(\mathrm{mSiO}_{2}\right)$ core-shell nanoparticles as the host and then $\mathrm{pH}$ - sensitive hydrazone linkage was connected to the mesoporous silica by means of chemical modification (Figure 2). Doxorubicin (DOX) was adopted as a typical anticancer drug to evaluate the controlled release. After drug loading, the as-prepared $\mathrm{Au}$ nanoparticles were used as nanoscopic caps to block the pore and inhibit the drug release. Because of the hydrazone bond, the drug-loading nanocomposites display acid-enhanced release with little premature release under neutral basic conditions ( $\mathrm{pH}$ 7.4). In addition, cell experiments (with HeLa cells) were carried out, thus revealing the negligible toxicity and good biocompatibility of the pure nanocomposites $\left(\mathrm{Fe}_{3} \mathrm{O}_{4} @ \mathrm{mSiO}_{2} @ \mathrm{Au}\right)$. Furthermore, DOX@ $\mathrm{Fe}_{3} \mathrm{O}_{4} @ \mathrm{mSiO}_{2} @ \mathrm{Au}$ reveals the pronounced cytotoxicity to HeLa cells, which is even similar to that of free DOX with concentrations up to $50 \mu \mathrm{gmL}^{-1}$.
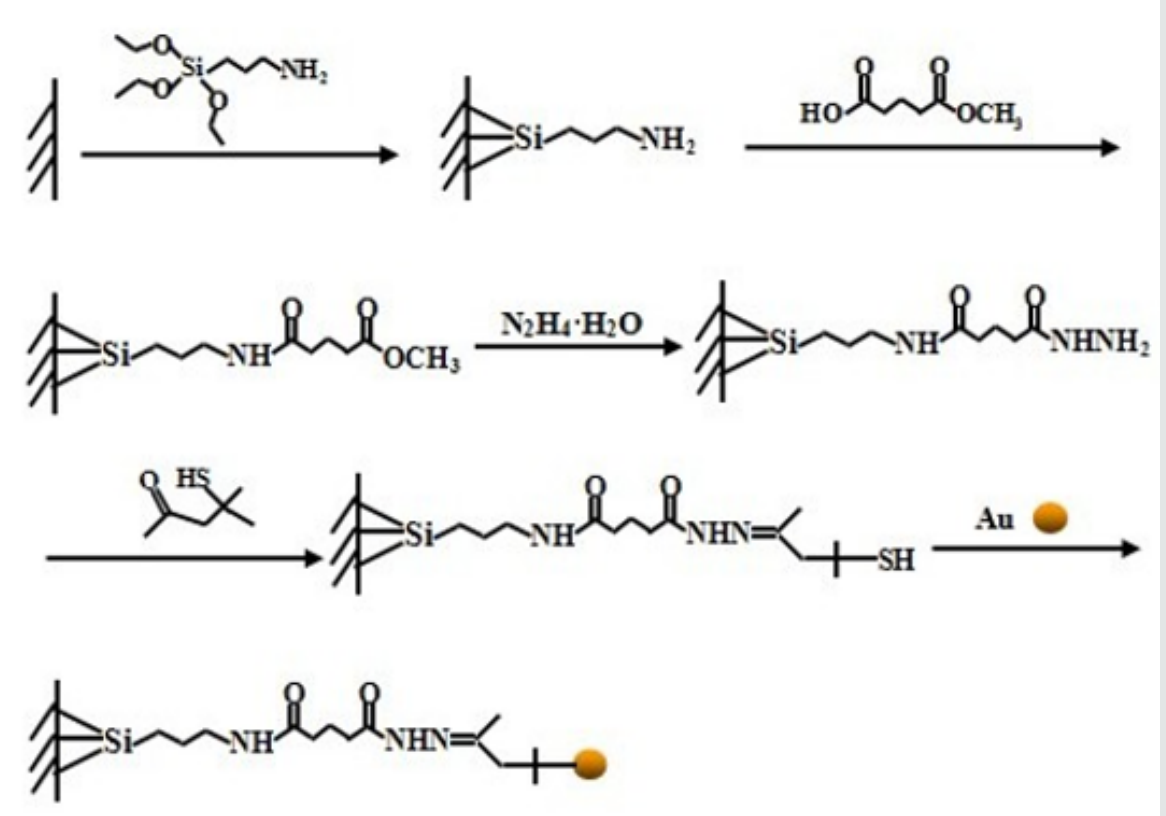

Figure 2: Reaction scheme of Au nanoparticles capped $\mathrm{Fe}_{3} \mathrm{O}_{4} @ \mathrm{mSiO}_{2}$.

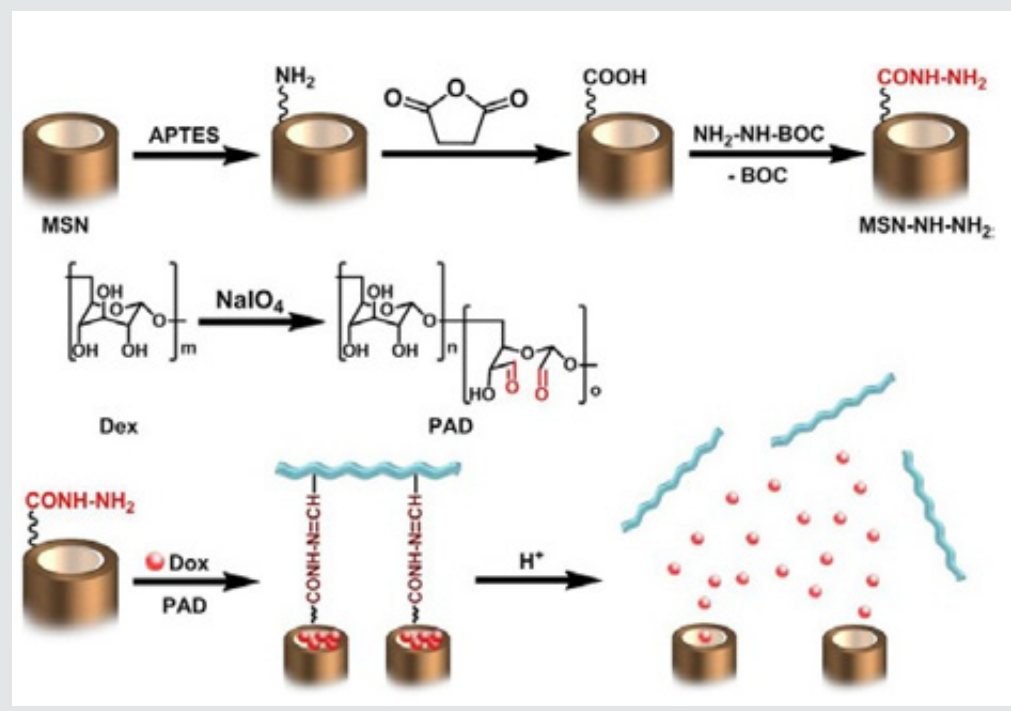

Figure 3: Fabrication of DOX@MSN-NH-N=C-PADs; and the intracellular release of DOX due to the low pH. 
Zhang et al. [6] applied a simple method to prepare the pH-sensitive dextran/MSNs based drug carriers in their work. As shown in Figure 3, MSNs were reacted with 3 aminopropyl triethoxysilane (APTES) to form the $\mathrm{MSN}-\mathrm{NH}_{2}$, which were further reacted with succinic anhydride to obtain $\mathrm{MSN}-\mathrm{COOH}$. Then tertbutyl carbazate was reacted with MSN-COOH to obtain MSN-NH$\mathrm{NH}_{2}$. After loading of DOX, three kinds of dextran dialdehydes (PADs) were applied to cap the pores of MSNs with hydrazone bond formed through the reaction between hydrazine of MSN-NH- $\mathrm{NH}_{2}$ and aldehyde of PADs. With the $\mathrm{pH}$ reduced from 7.4 to 5.5, the drug release rates were further accelerated. According to the results they suggested that drug carrier could entrap most of the DOX at pH 7.4 and burst release of the drugs in the weakly acidic environments, i.e. intracellular environment. Dai et al. [7] constructed a $\mathrm{pH}$ responsive drug delivery system for targeted tumor therapy based on hollow mesoporous silica nanoparticles (HMSNs). Hyaluronic acid (HA) molecules were employed as both blocking and targeting agents, which were anchored onto the surface of HMSNs through hydrazone bonds acting as pH-sensitive linkers. The fabricated targeted drug delivery systems could be effectively accumulated at tumor sites due to the targeting effect of HA molecules. And then, the linkers between HA blocking agents and HMSNs would be broken down triggered by the low $\mathrm{pH}$ value at tumor sites, leading to the removal of HA blocking agents and local anti-tumor drug release (Figure 4). Wong et al. [8] were synthesized a smart system in which DOX was conjugated to a zinc (II) phthalocyanine (ZnPc) through an acid-cleavable hydrazone linker. This azidocontaining conjugate was then anchored to the nano channels of an alkyne-modified mesoporous silica nanoparticle (MSN) system via copper(I)-catalyzed azide-alkyne cyclo addition. The acidsensitive hydrazone linker was cleaved under acidic conditions in PBS to release the DOX, and the rate of cleavage was promoted at lower $\mathrm{pH}$ values. These nanoparticles function as both nanophotosensitizers for Photodynamic therapy and as nanoplatforms for $\mathrm{pH}$ controlled drug release.

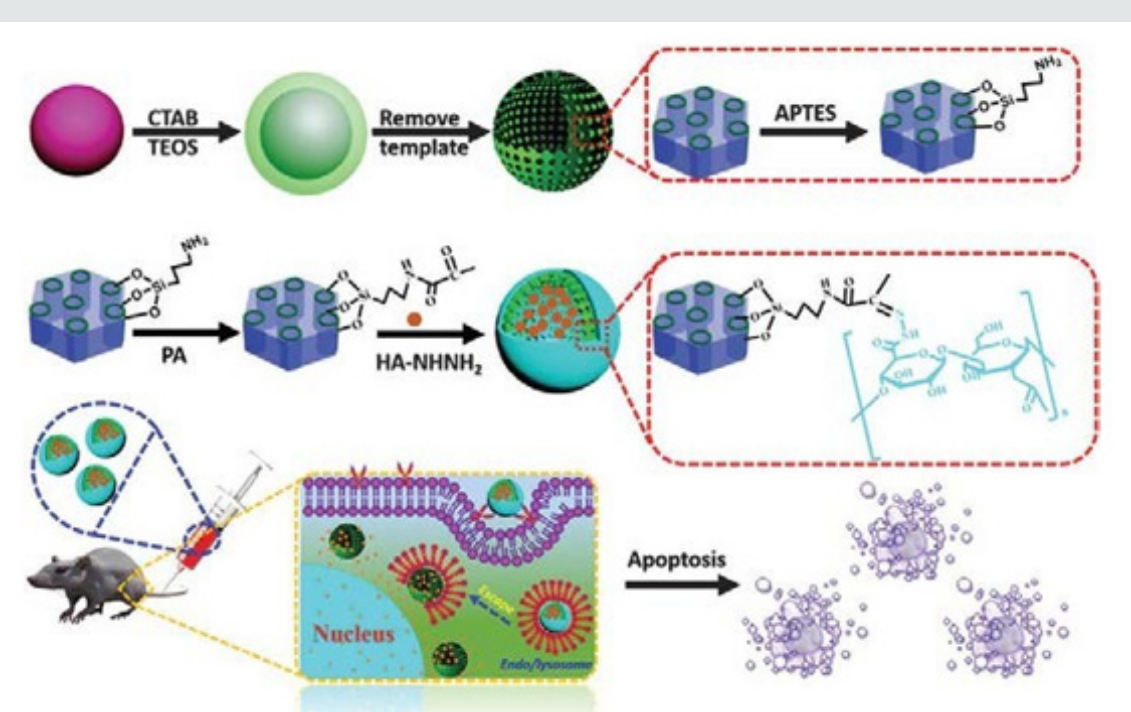

Figure 4: The construction of a HMSN-based drug delivery system for targeted tumor therapy [7].

Lin and coworkers [9] fabricated a $\mathrm{pH}$ and redox dualresponsive nanocarrier based on HA modified MSNs. DOX was conjugated to MSNs via hydrazone bonds, which can be cleaved in tumor tissue (acidic conditions). To improve specific cellular uptake and stability of nanocarriers, HA was equipped with an outer shell on the nanoparticle surface via a disulfide crosslinker. The results showed that the DOX can be controlled release from porous silica nanoparticles via $\mathrm{pH}$ and redox dual triggers. HA on the nanoparticle surface could lead to specific cellular uptake and the nanocarriers could targeted deliver to HeLa cells. These results demonstrated that the dual delivery system resulted in high local concentration inside tumor cells and lead to an effective cancer cell apoptosis, which may be promising for cancer therapeutic application. The conjugation of dexamethasone (DEX) onto modified-porous silica materials via a $\mathrm{pH}$ - responsive hydrazone bond has been reported to be highly efficient method to specifically deliver the DEX to diseased sites by Numpilai et al. [10]. In their paper, the impact of pore sizes, particle sizes and silanol contents on surface functionalization, drug loading and release behavior of porous silica materials conjugated with dexamethasone via pH-responsive hydrazone bond was investigated. The release of DEX from porous silica-DEX conjugates exhibited pH-dependent, sustained release, with the faster DEX release rate at an acidic $\mathrm{pH}$ of 4.5 being due to the hydrolysis of hydrazone bonds.

In order to improve the effects of medical therapy for cancer, Jiang and coworkers [11], prepared magnetic nanocomposites $\left(\mathrm{Fe}_{3} \mathrm{O}_{4} @ \mathrm{SiO}_{2}-\mathrm{NH}-\mathrm{NH}_{2}\right)$ as DOX carriers. In order to load DOX effectively, the surface of the carriers was further modified to make the surface with a large number of hydrazine bonds which can form a pH-sensitive bond (hydrazone bond) with DOX. However, DOXloaded carriers $\left(\mathrm{Fe}_{3} \mathrm{O}_{4} @ \mathrm{SiO}_{2}-\mathrm{DOX}\right)$ performed relatively poorer 
performance in terms of drug loading and releasing (the loading efficiency of DOX decreased from $67.33 \%$ to $42.15 \%$, while the releasing efficiency of DOX decreased from $66.16 \%$ to $62.23 \%$ within $72 \mathrm{~h}$ at $\mathrm{pH} 4.0$ ). Water-soluble tetrazolium salts (WST-1) assays in cancer cells (Hela) demonstrated that the $\mathrm{Fe}_{3} \mathrm{O}_{4} @ \mathrm{SiO}_{2}-$ DOX presented high anti-tumor activity. Xu et al. [12] report two types of stimuli-responsive nonporous silica prodrug nanoparticles by covalently linking anticancer drugs, namely camptothecin (CPT) and DOX, to silica precursors through the disulfide bond and hydrazine bond respectively. Dramatically enhanced release of the entrapped anticancer drugs was observed upon exposure of these prodrug nanoparticles to glutathione rich or acidic conditions similar to the cellular microenvironment of cancer cells. In vitro drug release studies indicated that the prodrug nanoparticles effectively inhibited the proliferation of cancer cells and showed an impressive anticancer efficacy. Moreover, the combined cancer chemotherapy using both CPT and DOX delivered by the silica prodrug nanoparticles was studied, indicating a promising combinatorial therapeutic effect.

\section{Acetal Linker}

The acetal linkers are stable under neutral conditions $\mathrm{pH}$ 7.4), while can be cleaved under acidic conditions. Chen et al. [13] constructed drug delivery systems by immobilizing acetals on the surface of mesoporous silica, and then coupling to ultra-small lanthanide doped upconverting nanoparticle, which act as a gate keeper. The anti-cancer drug DOX, is thus locked in the pores, and its burst release can be achieved under acidic environment on account of the hydrolyzation reactions of acetals. The resulted system was believed to be able to efficiently transport DOX into the cancer cells, released rapidly in lysosomes and endosomes due to the acidic situation. The nanogated drug release system was highly efficacious for cancer therapy both in vitro and in vivo. After intravenous injection into the murine model, such nanocomposite, which accumulated in several organs, was degraded into apparently nontoxic products within a few days. Despite the enhanced therapeutic through the so-called Enhanced Permeability and Retention (EPR) effect, which was visualized by Magnetic Resonance Imaging (MRI). Yang and coworkers [14] grafted an acid-labile acetal linker 3,9bis(3- aminopropyl)-2,4,8,10- tetraoxaspiro[6] undecane (BATU) to mesoporous silica surface modified with carboxyl groups via the EDC/NHS process. Then an antineoplastic drug DOX is filled into the mesopores of functional mesoporous silica with the surface coated with poly(N-succinimidylacrylate) (PSA). They found that the polymer coating layer effectively blocked the pore channels and prevented the entrapped antineoplastic drug DOX from premature release at physiological $\mathrm{pH}$. Under the acidic conditions, DOX could be rapidly released as a result of the disassociation of polymer coating layer. Importantly, the massive drug molecules were able to be released in mild acidic conditions $(\mathrm{pH}=5-6)$.
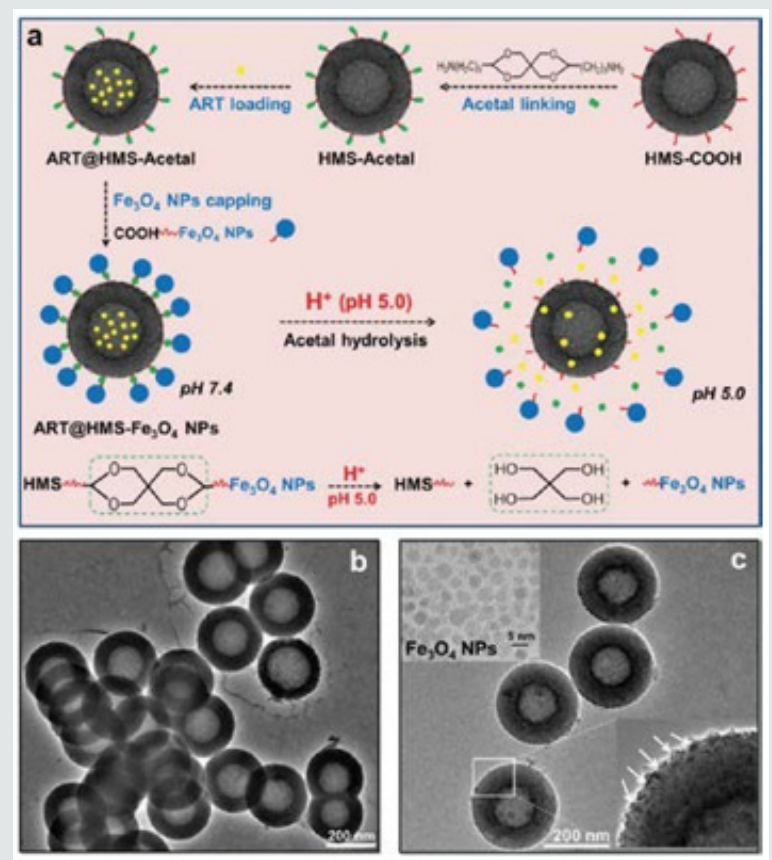

Figure 5: (a) Illustration of the pH-responsive ART@HMS- $\mathrm{Fe}_{3} \mathrm{O}_{4}$ nano system. (b) TEM images of the as-prepared ART@HMSacetal and (c) ART@HMS- $\mathrm{Fe}_{3} \mathrm{O}_{4}$. The inset of (c) is the TEM image of the as-prepared $\mathrm{Fe}_{3} \mathrm{O}_{4}$ nanoparticles [15].

Fu et al. [15] developed, a smart and pH-switched prodrug/ procatalyst co-delivery nanosystem which is non-toxic toward normal cells and is inert during its delivery in the vasculature, while responsively functions in acidic lysosomes inside cancer cells
(Figure 5). They chose HMSNPs as nanocarriers and encapsulated hydrophobic artemisinin (ART) into HMSNPs (defined as ART@ HMS) to improve their solubility and bioavailability. To guarantee zero leakage of ART during their delivery, $\mathrm{Fe}_{3} \mathrm{O}_{4}$ NPs (ca. $4 \mathrm{~nm}$ ) 
were grafted onto the pore outlets of ART@HMS via acid cleavable acetal linkers (defined as ART@HMS- $\mathrm{Fe}_{3} \mathrm{O}_{4}$ ). They reported, "zero release" in the non-target areas of the vasculature and extracellular environment ( $\mathrm{pH}$ 6.8-7.4), and "controlled release" in the target areas of lysosomes inside cancer cells (pH 3.8-5.0). Liu et al. [16] reported the controlled release of guest molecules from mesoporous silica particles by using acid-labile acetal group linked gold nanoparticles as pH-responsive capping agents. The guest molecules were blocked from the hybrid materials at neutral $\mathrm{pH}$ and released at lower $\mathrm{pH}$. The release profile is strongly dependent on the cleavage of the acetal linker at different pH's. The results make the system reported a promising candidate in the formulation of a $\mathrm{pH}$-sensitive vehicle for in vivo delivery of therapeutic agents to low pH tissues, such as tumors and inflammatory sites (Figure 6).

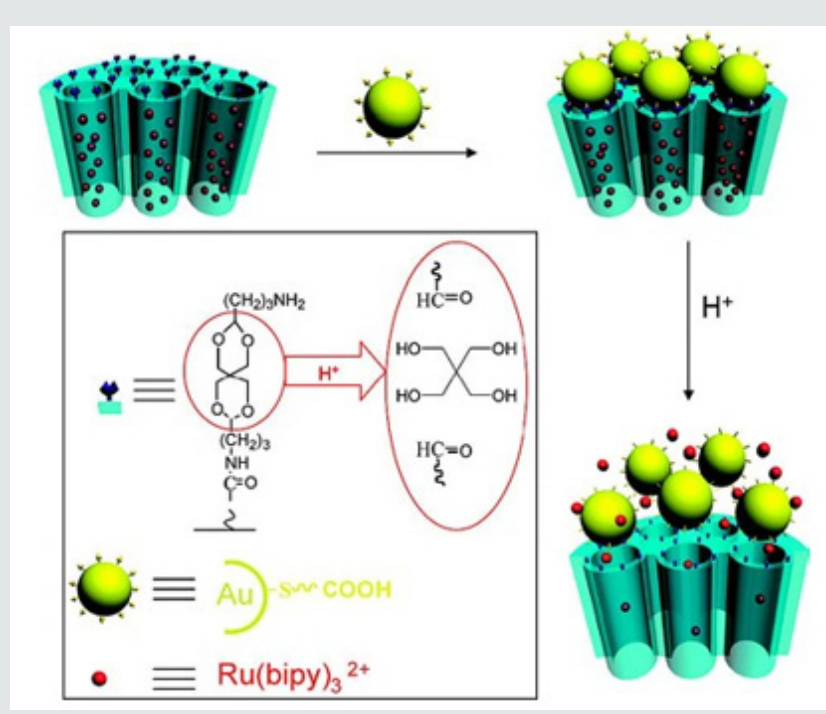

Figure 6: Schematic illustration of $\mathrm{pH}$-responsive nanogated system [16]

Martínez-Carmona et al. [17] have developed a multifunctional nanodevice featuring selectivity towards human osteosarcoma cells and pH-responsive antitumor drug delivery capability. This innovative nanodevice is based in DOX-loaded MSNs nanoplatforms where different building blocks are assembled:

I. A polyacrylic acid (PAA) polymeric shell, anchored via an acid cleavable linker, to prevent premature cargo release and provide the nano system of pH-responsive capability.

II. A targeting ligand consisting in the lectin Con A grafted to PAA, to increase the selectivity towards cancer cells whereas significantly preserving the viability of healthy cells.

This multifunctional nano system exhibits a noticeable higher internalization degree into human osteosarcoma cells (HOS), overexpressing sialic acids (SA), compared to healthy preosteo blast cells (MC3T3-E1). Moreover, the results indicate that small DOX loading $\left(2.5 \mu \mathrm{gmL}^{-1}\right)$ leads to almost $100 \%$ of osteosarcoma cell death in comparison with healthy bone cells, which significantly preserve their viability.

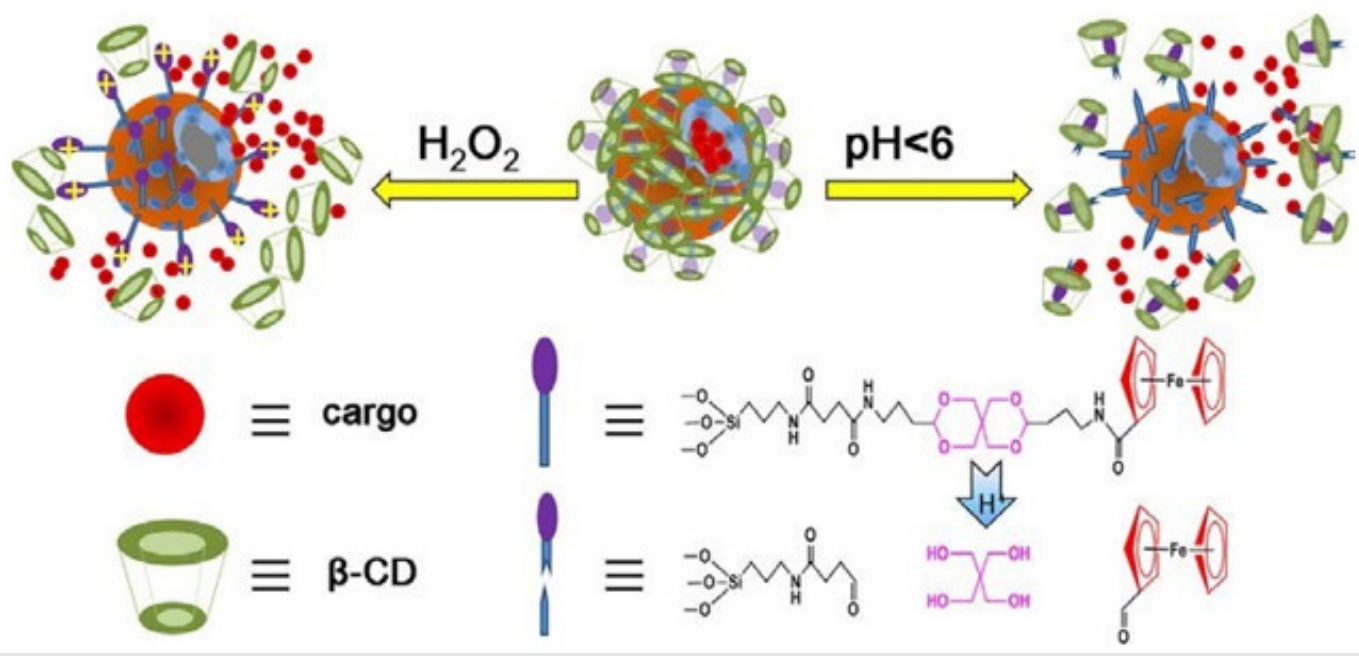

Figure 7: Schematic diagram of the release of R6G from mechanized HMSNs-S1 [18]. 
Zhu and coworkers [18] designed a pH and redox dualresponsive supramolecular nanovalve system based on the hostguest interaction of $\beta$-cyclodextrins/ferrocenyl moiety $(\beta-\mathrm{CD} / \mathrm{Fc})$ inclusion complexes and an acetal linker to control release of cargos Rhodamine 6G (R6G) as model drug molecule was encapsulated in HMSNs-S1, at pH 7.4, almost no leakage was observed, while the R6G was released at acidic environment (Figure 7). A pH-sensitive drug release system using acetalated-dextran as valves was designed to manipulate smart intracellular release of anticancer drugs by Zhe et al. [19]. Dextran was grafted onto the exterior of MSNs through a click reaction, and followed by acetylation to generate the final carriers of MSN-Dex-Ac. The hydrophobic Dex-Ac would act as valves on the MSNs surface to block the entrapped drugs inside the MSNs pores. While under acidic conditions mimicking the microenvironment of endosomal/lysosomal compartments, the valves could be opened by acetal hydrolysis to recover the acetalateddextran to its hydrophilic state, resulting in fast drug release. In vitro drug release profile clearly showed that DOX release was restricted at $\mathrm{pH} 7.4$ by the valves, while it was accelerated under acidic conditions.

\section{Imine Linker}

(a)

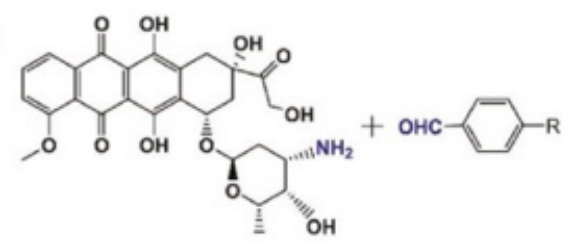

(b)
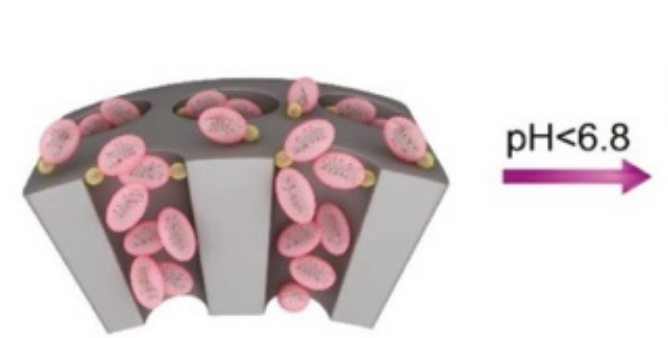
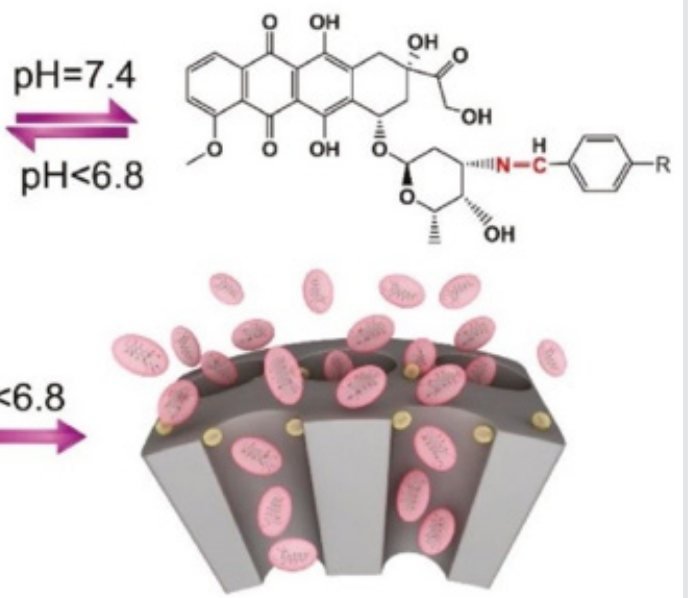

Figure 8: a) The dynamic interaction between DOX and benzaldehyde via pH-sensitive benzoic-imine bond. b) Schematic illustration of the drug DOX-self-gated MSNs with $\mathrm{pH}$ - responsive drug release property.

The benzoic-imine bond is highly pH-sensitive, and the bond would break under acidic conditions such as tumor tissue or cells ( $\mathrm{pH}<6.8$ ), thus leading to a switchable and simultaneous delivery of siRNA and anticancer drugs at desired parts. The pH-labile imine bond is very attractive for the construction of controlled drug delivery since primary amines are existed widely in drug molecules. For example, the research group of Zeng [20] present a facile drug- self-gated MSNs as anticancer nanoplatform via $\mathrm{pH}$ sensitive dynamic benzoic-imine covalent bond. The major aim in this study is to employ the drug itself as a pH-sensitive "gatekeeper" to minimize the potential risks of auxiliary capping agents that have been generally used in previous systems. In their design, DOX was used as the drug cargo as well as the "gatekeeper." A benzaldehydefunctionalized MSNs was loaded with DOX and then gated by DOX on pore outlets via the formation of benzoic-imine covalent bond between DOX and benzaldehyde groups. In case of being at weak acidic tumor tissue/cells $(\mathrm{pH}<6.8)$, the loaded DOX could be on-demand released due to the $\mathrm{pH}$-induced hydrolyzation of benzoic-imine bonds (Figures $8 \mathrm{a} \& \mathrm{8b}$ ). This is the first drug-selfgated system that does not require complex capping agents and also possesses switchable drug release behavior triggered by an internal biological stimulus.

For the design of the dual-controlled release system Zhao and coworkers [21] chosed imine group and the complex of $\beta$-CD with azobenzene derivative, for $\mathrm{pH}$ and light trigger respectively. The system was prepared by surface modification of MSNs (MCM-41) with imine group and $\beta-C D$ and azobenzene complex which were used for $\mathrm{pH}$ and light controlled delivery, respectively. The surface modification efficiently blocked the cargo release in $\mathrm{pH}=7.0 \mathrm{PBS}$ without $365 \mathrm{~nm}$ UV irradiation. Plenty cargos would be delivered if both factors of acidic environment ( $\mathrm{pH}=5.0)$ and UV light (365nm) irradiation were satisfied, meanwhile only few cargos were released when one factor was satisfied. Chen et al. [22] prepared MSN based drug carrier to load anti-cancer drug DOX and covered by mono6-deoxy-6-EDA-b-cyclodextrine $\left(\beta-\mathrm{CD}-\mathrm{NH}_{2}\right)$ to block the pores through $\mathrm{pH}$-sensitive boronate ester bond. And the carriers were then coated with methoxy poly(ethylene glycol) (mPEG) through another pH-sensitive benzoic imine bond. mPEG leaving studies, in vitro cellular uptake studies and the flow cytometry analysis, proved that carriers was "stealthy" at pH 7.4, but could be "activated" for cytophagy by cancer cells in weakly acidic tumor tissues $(\mathrm{pH}$ 6.5) due to the departure of mPEG. $\beta$-CD- $\mathrm{NH}_{2}$ leaving studies, the in vitro drug release studies and the in vitro cytotoxicity studies proved that boronate ester bond linking MSN and $\beta-\mathrm{CD}-\mathrm{NH}_{2}$ was stable at both pH 7.4 and 6.5 but could be hydrolyzed intracellular to release DOX for cellular apoptosis due to the lower $\mathrm{pH}$ (5.0). 
Cheng and coworkers [23] fabricated a facile and concise codelivery system using the anticancer drug DOX as the drug cargo as well as the gatekeeper of their system. The pore outlets of the MSNs were gated by the drug itself through the formation of a benzoicimine covalent bond between the amino groups of DOX and the benzaldehyde groups of benzaldehyde-functionalized MSNs under weakly alkaline conditions. Moreover, another distinct advantage of this co-delivery system is that the anticancer drug itself serves as a "gatekeeper", which can minimize potential risks brought by other extra capping agents (Figure 9) Zhang et al. [24] reported a novel "stealthy" chitosan (CHI)/MSN complex drug delivery system with tumor-triggered intracellular drug release properties. In this system, MSN was used as the nano-container for the loading of the anticancer drug DOX and was covered by $\mathrm{CHI}$ to block the pores through redox-sensitive disulfide bonds. Then mPEG was grafted to the surface of the nanoparticles via a $\mathrm{pH}$-sensitive benzoic imine linker. The system was "stealthy" in physiological environments at pH 7.4 but was "activated" in weakly acidic environments, such as that found in solid tumor tissues, due to hydrolysis of the benzoic imine and the departure of the mPEG shields (Figure 10).

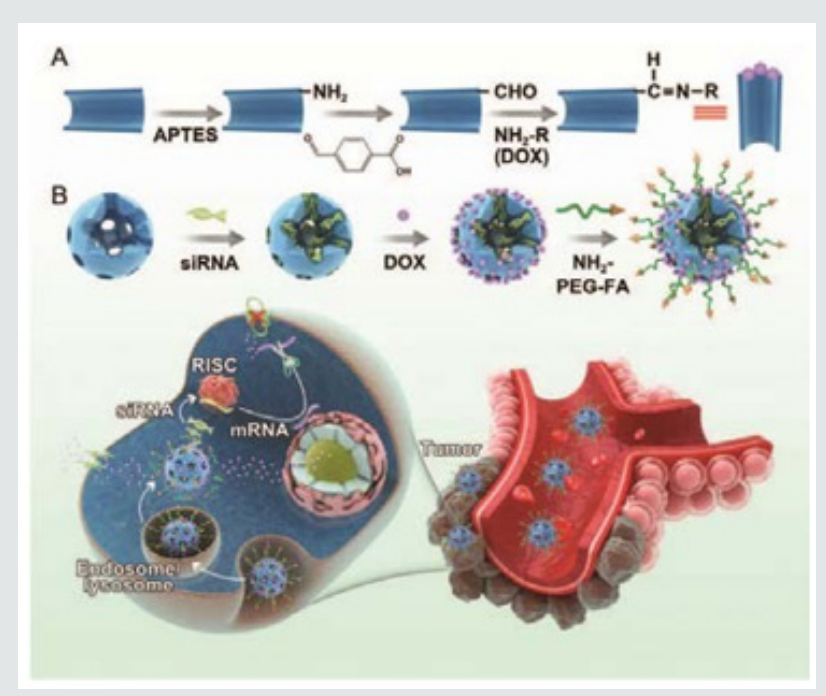

Figure 9: Schematic illustration of (A) the synthesis route to MSNs-CHO and the dynamic interaction between doxorubicin (DOX) and benzaldehyde via a pH-sensitive benzoic-imine bond. (B) The synthesis route to MSNs-siRNA@DOX-PEG-FA and the combined chemo/gene targeted therapy of tumors [23].

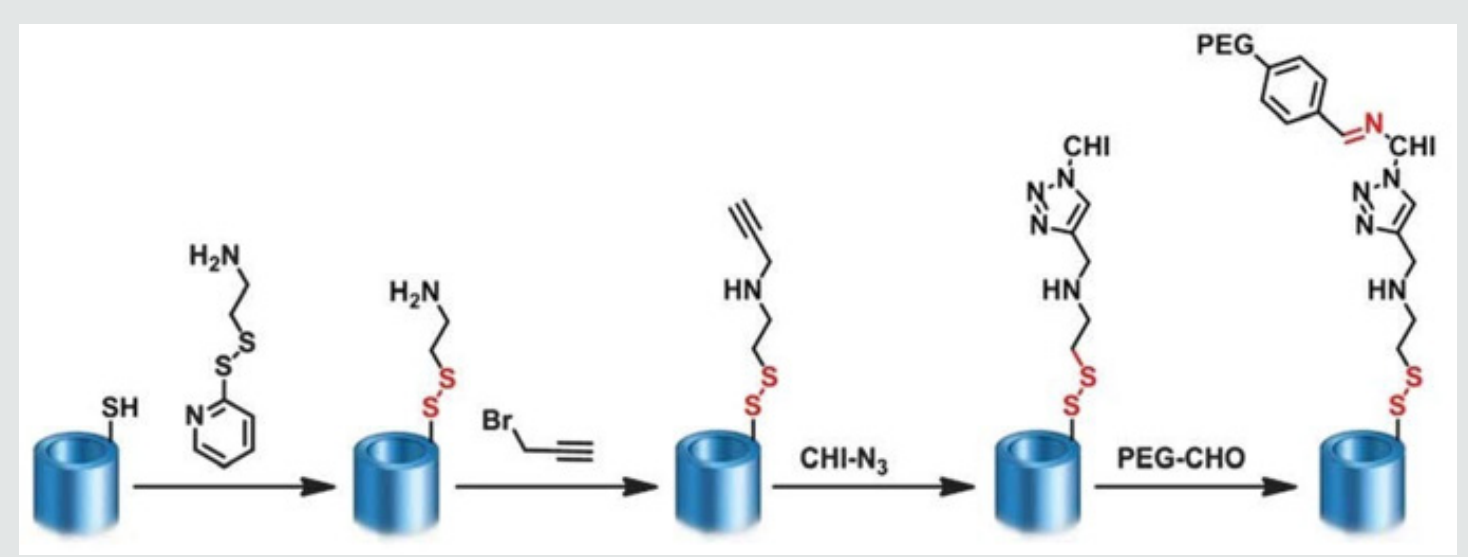

Figure 10: Fabrication route of DOX@MSN-SS-CHI-PEG [24].

\section{Boronic-Ester Linker}

Liu and coworkers [25] report a novel multifunctional tumor microenvironment cascade $\mathrm{pH}$ stimulus triggering drug delivery system based on HMSNs for tumor therapy. HMSNs serve as drug reservoirs for loading model anticancer drug of DOX. The $\beta$-CD was employed as gatekeeper of HMSNs due to its appropriate molecular size and good biocompatibility. Briefly, HMSNs were firstly modified with boronic acid molecules for anchoring $\beta-C D$ onto HMSNs with pH sensitive boronic acid catechol ester bonds ( $\mathrm{pH}$ 5.0), leading to encapsulation of DOX within mesopores of HMSNs; Then polyethylene glycol (PEG) was grafted to adamantane (Ada) through weak $\mathrm{pH}$ sensitive benzoic-imine bonds $(\mathrm{pH} 6.8$ ) (Figure 11). PEG protective layer could efficiently suppress nonspecific protein adsorption and cellular uptake, and thus prolong the blood circulation of the system. After the system reaching tumor site via EPR effect, the tumor microenvironment $\mathrm{pH}$ signal 
(6.8) would break down benzoic-imine bonds to release PEG layer for improving cellular uptake. Then, the endosome/lysosome $\mathrm{pH}$ signal (5.0) would further hydrolyze boronic acid-catechol ester

bonds and dissociate gatekeeper of $\beta-\mathrm{CD}$, leading to intracellular drug delivery for inducing of cell apoptosis and inhibition of tumor growth.

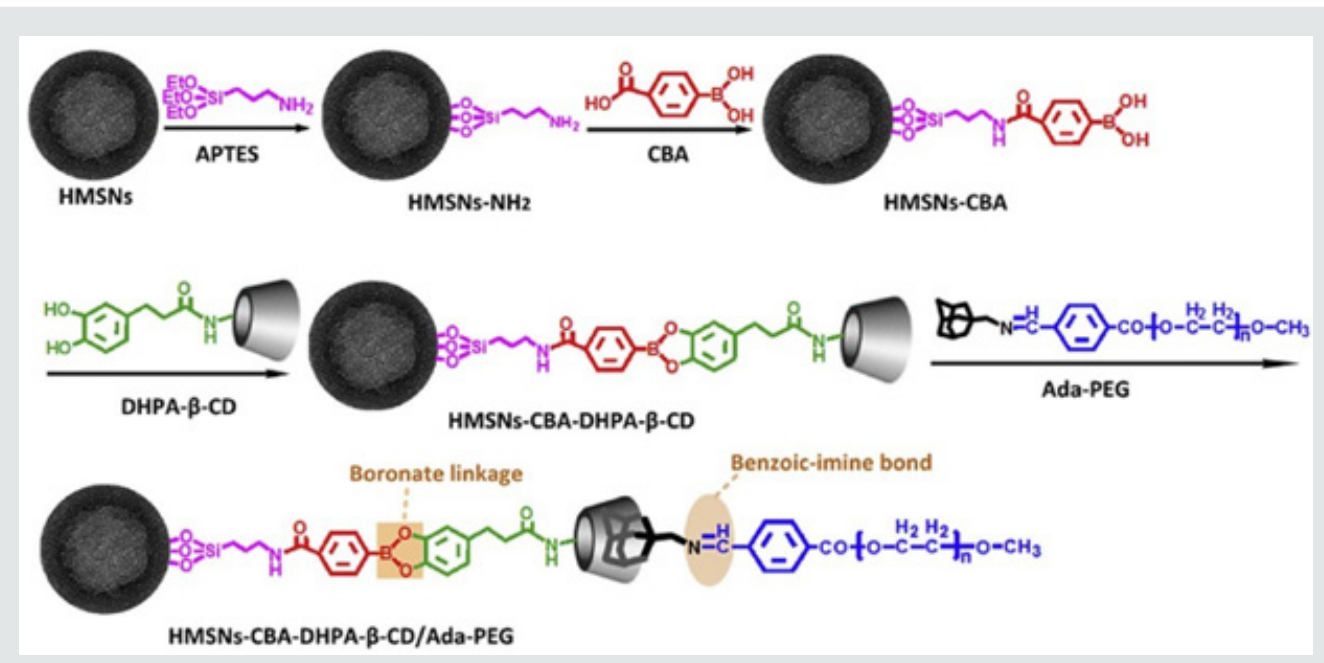

Figure 11: Fabrication of cascade pH-responsive HMSNs-based drug delivery system.

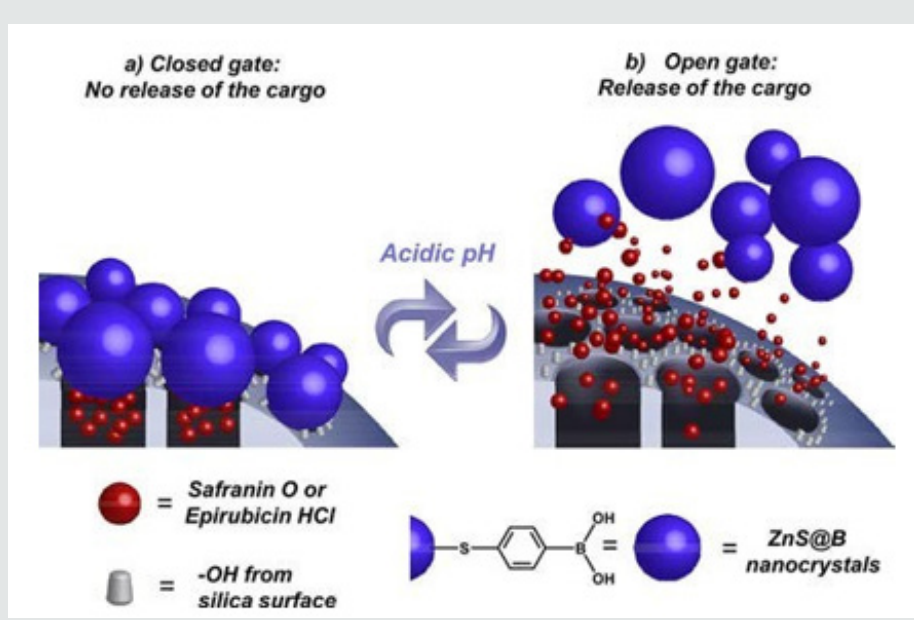

Figure 12: (a) MSNs loaded with safranin O (S2-Saf) or epirubicin (S2-Epi) and capped with boronic-acid-coated ZnS nanocrystals (ZnS@B) (closed gate, no release of the cargo); (b) cargo release achieved by hydrolysis of boronate esters, formed between the boronic acid moieties of nanocrystals and silanols $(-\mathrm{OH})$ from the silica surface at acidic pH (open gate, release of the cargo) [26].

Salinas et al. [26] have demonstrated the possibility of directly attaching non-toxic ZnS-based nanocrystals as pore blockers on MSNs to efficiently control the release of uploaded molecules, such as fluorophore safranin 0 or anticancer drug epirubicin hydrochloride, at acidic pHs $(\leq 5.2)$. Biocompatible ZnS-based nanocrystals capped with 4-mercaptophenylboronic acid (ZnS@B) have been size-designed as excellent $\mathrm{pH}$ responsive gatekeepers on MSNs which encapsulate fluorophore safranin O (S2-Saf) or anticancer drug epirubicin hydrochloride (S2- Epi) for delivery applications in cancer cells. In this novel hybrid system, the gate mechanism consists of reversible $\mathrm{pH}$-sensitive boronate ester moieties linking the nanocrystals directly to the alcohol groups from silica surface scaffold, avoiding tedious intermediate functionalization steps. The $\sim 3 \mathrm{~nm}$ size of the ZnS@B nanocrystals was tailored to allow efficient sealing of the pore voids and achieve a "zero premature cargo release" at neutral pH (7.4). The system selectively released the cargo in acidic conditions (pH 5.4 and 3.0) because of the hydrolysis of the boronate esters, which unblocked the pore voids (Figure 12). Tan et al. [27] presented in their paper, a glucose and $\mathrm{pH}$-responsive release system based on polymeric network capped MSNs. The PAA brush on MSN was obtained through the surface- initiated atom transfer radical polymerization (SI-ATRP) of t-butyl acrylate and the subsequent hydrolysis of the ester bond. Then the PAA was glycosylated with glucosamine to obtain P(AA- AGA). To block the pore of silica, the P(AA-AGA) chains were cross-linked through the formation of boronate esters between 4,4-(ethylenedicarbamoyl)-phenylboronic acid (EPBA) and the hydroxyl groups of P(AA-AGA). The boronate esters disassociated in the presence of glucose or in acidic conditions, which lead to opening of the mesoporous channels and the release 
of loaded guest molecules. The rate of release could be tuned by varying the $\mathrm{pH}$ or the concentration of glucose in the environment. The combination of two stimuli exhibited an obvious enhanced release capacity in mild acidic conditions ( $\mathrm{pH}$ 6.0).

\section{Conclusion}

In the last decades smart nanostructured materials have received numerous interests to improve drug delivery performance of classic administration routes. Various drug delivery approaches can be used to maximize therapeutic efficacy. MSNs have received numerous interests for drug delivery systems due to their remarkable features such as; high physicochemical and biochemical stability, tunable mesopore size, biocompatibility, large surface areas and pore volumes. Although unmodified MSNs have unique properties, their inherent hydrophobicity and single physical loading ability have largely restricted their biomedical drug delivery applications. To overcome this obstacle, surface modification of MSNs is necessary to develop smart multifunctional MSNs coated with tumor microenvironment responsive gatekeepers.

\section{References}

1. Jafari S, Derakhshankhah H, Alaei L, Fattahi A, Varnamkhasti BS, et al. (2019) Mesoporous silica nanoparticles for therapeutic/diagnostic applications. Biomedicine \& Pharmacotherapy 109: 1100-1111.

2. Prabha S, Durgalakshmi D, Aruna P, Ganesan S (2019) Influence of the parameters in the preparation of silica nanoparticles from biomass and chemical silica precursors towards bioimaging application. Vacuum 160: 181-188.

3. Liang CL, Xiang Q Cui WM, Fang J, Sun NN, et al. (2018) Subchronic Oral Toxicity of Silica Nanoparticles and Silica Microparticles in Rats. Biomed Environ Sci 31(3): 197-207.

4. Zhang Y, Wang W, Zhang Y, Cheng M, Wu Q, et al. (2018) Three-in-One Multifunctional Gatekeeper Gated Mesoporous Silica Nanoparticles for Intracellular pH-Activated Targeted Cancer Therapy. ACS Applied Bio Materials 1(3): 572-580.

5. Cui L, Lin H, Yang C, Han X, Zhang T, et al. (2014) Synthesis of Multifunctional $\mathrm{Fe}_{3} \mathrm{O}_{4} @ \mathrm{mSiO}_{2} @ A u$ Core-Shell Nanocomposites for pHResponsive Drug Delivery. European Journal of Inorganic Chemistry, pp. 6156-6164.

6. Zhang M, Liu J, Kuang Y, Li Q Zheng DW, et al. (2017) Ingenious pHsensitive dextran/mesoporous silica nanoparticles-based drug delivery systems for controlled intracellular drug release. International journal of biological macromolecules 98: 691-700.

7. Dai L, Zhang Q, Shen X, Sun Q, Mu C, et al. (2016) A pH-responsive nanocontainer based on hydrazone-bearing hollow silica nanoparticles for targeted tumor therapy. Journal of Materials Chemistry B 4(26): 4594-4604.

8. Wong RCH, Ng DKP, Fong WP, Lo PC (2017) Encapsulating pH-Responsive Doxorubicin- Phthalocyanine Conjugates in Mesoporous Silica Nanoparticles for Combined Photodynamic Therapy and Controlled Chemotherapy. Chemistry- A European Journal 23(65): 16505-16515.

9. Lin JT, Du JK, Yang YQ, Li L, Zhang DW, et al. (2017) pH and redox dual stimulate-responsive nanocarriers based on hyaluronic acid coated mesoporous silica for targeted drug delivery. Mater Sci Eng C Mater Biol Appl 81: 478- 484.

10. Numpilai T, Witoon T, Chareonpanich M, Limtrakul J (2017) Impact of physicochemical properties of porous silica materials conjugated with dexamethasone via $\mathrm{pH}$-responsive hydrazone bond on drug loading and release behavior. Applied Surface Science 396: 504-514.
11. Jiang W, Wu J, Shen Y, Tian R, Zhou S, et al. (2016) Synthesis and Characterization of Doxorubicin Loaded pH-Sensitive Magnetic CoreShell Nanocomposites for Targeted Drug Delivery Applications. Nano 11(11): 1650127.

12.Xu Z, Liu S, Kang Y, Wang M (2015) Glutathione- and pH-responsive nonporous silica prodrug nanoparticles for controlled release and cancer therapy. Nanoscale 7(13): 5859-5868.

13. Chen Y, Ai K, Liu J, Sun G, Yin Q, et al. (2015) Multifunctional envelopetype mesoporous silica nanoparticles for $\mathrm{pH}$-responsive drug delivery and magnetic resonance imaging. Biomaterials 60: 111-120.

14. Yang $\mathrm{K}$, Luo H, Zeng M, Jiang Y, Li J, et al. (2015) Intracellular pHTriggered, Targeted Drug Delivery to Cancer Cells by Multifunctional Envelope-Type Mesoporous Silica Nanocontainers. ACS Appl Mater Interfaces 7(31): 17399-17407.

15. Fu J, Zhu Y (2017) Lysosomes activating chain reactions against cancer cells with a pH-switched prodrug/procatalyst co-delivery nanosystem. Journal of Materials Chemistry B(5): 996-1004.

16. Liu R, Zhang Y, Zhao X, Agarwal A, Mueller LJ, et al. (2010) pH-Responsive Nanogated Ensemble Based on Gold-Capped Mesoporous Silica through an Acid-Labile Acetal Linker. Journal of the American Chemical Society 132(5): 1500-1501.

17. Martínez-Carmona M, Lozano D, Colilla M, Vallet-Regí M (2018) Lectinconjugated pH-responsive mesoporous silica nanoparticles for targeted bone cancer treatment. Acta Biomaterialia 65: 393-404.

18. Zhu X, Wang CQ (2016) pH and redox-operated nanovalve for sizeselective cargo delivery on hollow mesoporous silica spheres. J Colloid Interface Sci 480: 39-48.

19. Lin Z, Li J, He H, Kuang H, Chen X, et al. (2015) Acetalated-dextran as valves of mesoporous silica particles for $\mathrm{pH}$ responsive intracellular drug delivery. RSC Advances 5: 9546- 9555.

20.Zeng X, Liu G, Tao W, Ma Y, Zhang X, et al. (2017) A Drug-Self-Gated Mesoporous Antitumor Nanoplatform Based on pH-Sensitive Dynamic Covalent Bond. Advanced Functional Materials 27: 1605985.

21. Zhao J, He Z, Li B, Cheng T, Liu G (2017) AND logic-like pH- and lightdual controlled drug delivery by surface modified mesoporous silica nanoparticles, Materials science \& engineering. C. Mater Sci Eng C Mater Biol Appl 73: 1-7.

22. Chen H, Kuang Y, Liu R, Chen Z, Jiang B, et al. (2018) Dual-pH-sensitive mesoporous silica nanoparticle-based drug delivery system for tumortriggered intracellular drug release. Journal of Materials Science 53(15): 10653-10665.

23. Cheng W, Liang C, Wang X, Tsai Hi, Liu G, et al. (2017) A drug-self- gated and tumor microenvironment-responsive mesoporous silica vehicle: "four-in-one" versatile nanomedicine for targeted multidrug-resistant cancer therapy. Nanoscale 9(43): 17063-17073.

24. Zhang M, Liu J, Kuang Y, Li Q Chen H, et al. (2016) “Stealthy” chitosan/ mesoporous silica nanoparticle based complex system for tumortriggered intracellular drug release. Journal of Materials Chemistry B 4(19): 3387-3397.

25. Liu J, Luo Z, Zhang J, Luo T, Zhou J, et al. (2016) Hollow mesoporous silica nanoparticles facilitated drug delivery via cascade $\mathrm{pH}$ stimuli in tumor microenvironment for tumor therapy. Biomaterials 83: 51-65.

26. Salinas Y, Hoerhager C, Garcia-Fernandez A, Resmini M, Sancenon F, et al. (2018) Biocompatible Phenylboronic-Acid-Capped ZnS Nanocrystals Designed as Caps in Mesoporous Silica Hybrid Materials for on-Demand pH-Triggered Release in Cancer Cells. ACS applied materials \& interfaces 10(40): 34029-34038.

27. Tan L, Yang MY, Wu HX, Tang ZW, Xiao JY, et al. (2015) Glucose- and pHResponsive Nanogated Ensemble Based on Polymeric Network Capped Mesoporous Silica. ACS applied materials \& interfaces 7(11): 6310-6316. 


\section{CC (i) This work is licensed under Creative Submission Link: Submit Article}

DOI: $10.32474 /$ DDIPIJ.2019.02.000146

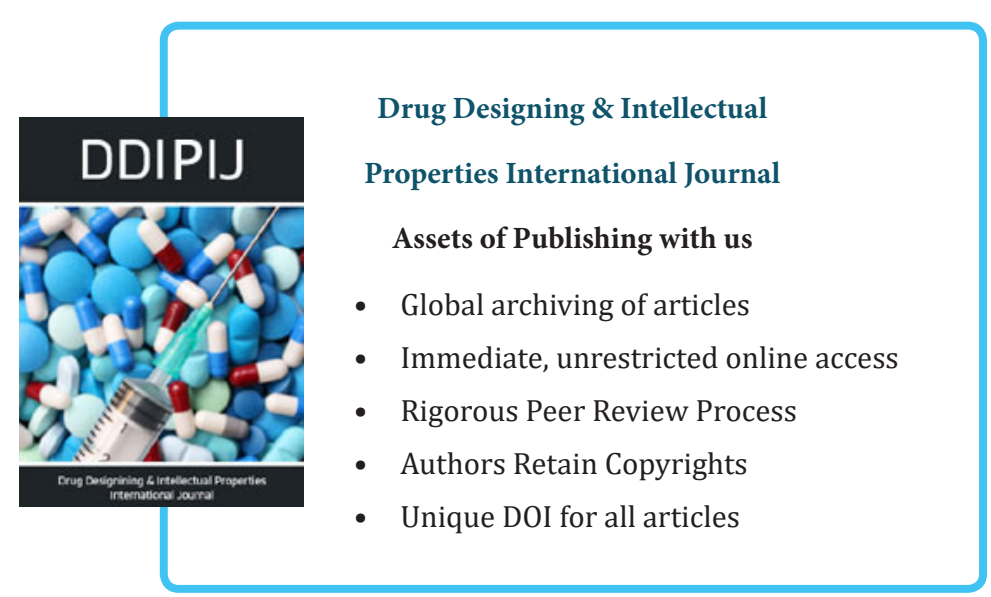

